\section{Hardness Measurements of Silicon Nanospheres Yield Values Four Times that for Bulk Silicon}

Recently, a team of researchers from the University of Minnesota and Los Alamos National Laboratory directly determined the mechanical response of single nanospheres of silicon with hardness up to $50 \mathrm{GPa}$, which is $4 \times$ greater than that normally expected for bulk silicon (12 GPa).

As reported in the June issue of the Journal of Mechanics and Physics in Solids, W.W. Gerberich, M.I. Baskes, R. Mukherjee and colleagues first synthesized these monodispersed silicon particles by injecting vapor-phase silicon tetrachloride into an argon hydrogen plasma using a modified hypersonic plasma particle deposition technique. These particles were then transported to an aerodynamic lens system in a streamline under the influence of fluid drag and particle inertia. Lines of nanoparticles were deposited across a sapphire wafer mounted on a computer-controlled substrate translation system.

The researchers began their study by performing an atomistic simulation of a 12-nm-diameter silicon nanosphere and nanoindentation measurements of a 38.6-nm-diameter silicon nanosphere. The atomistic simulation of the 12-nm-diameter silicon nanosphere, which used a modified embedded atom method (MEAM) potential, showed that no apparent dislocation nucleation occurred when two stiff platens compressed it. The unloaded nanosphere had only a $\sim 5$ change in size in the direction of the surface contact, due to phase transformation and flow of $\mathrm{Si}$ atoms toward the outer regions of the nanosphere, in agreement with a geometric (perfectly plastic) contact. In contrast, the nanoindentation measurements on the larger silicon nanosphere showed staircase yielding events, which were attributed to the nucleation of contact loops at the contact edges that then move down or up a glide cylinder. The researchers indicated that the differences between the simulation of the $12 \mathrm{~nm}$ nanosphere and the measurements on the $38.6 \mathrm{~nm}$ nanosphere may be due to the inability of dislocation loops to nucleate in small enough volumes.

Subsequently, spheres from $40 \mathrm{~nm}$ to $100 \mathrm{~nm}$ in diameter were repeatedly compressed. For these larger nanospheres, the researchers believe that plastic deformation is accomplished by dislocation nucleation. For these, it is shown that reverse plastic strain increases as the particle size decreases. For 38.6-nm-diameter silicon spheres, these particles showed as much as $48.6 \%$ strain reversal after they were loaded up to $70 \mu \mathrm{N}$ and then unloaded. From these observations, the researchers proposed that this strain reversal is due to high dislocation densities within the particles and that these high densities also effectively work to harden the particle. For future studies, the researchers plan to verify this finding by transmission electron microscopy to confirm particle shape, size, and examine the residual dislocation array after indentation. The researchers indicated that superhard nanospheres may have potential applications in chemical-mechanical polishing or planarization of microelectronics and read-write heads of disk drives as well as for hardening surfaces of microelectromechanical systems devices.

KINSON C. KAM

\section{Biomimetic Strategy for Antifouling Materials Developed from Mussel Adhesive Protein Mimetic Polymers}

The use of surface-modification techniques with poly(ethylene glycol) (PEG) has been shown to prevent protein, cell, and organism fouling at the interfaces with biological tissues. PEG is a biocompatible polymer and can be immobilized on the surfaces by the specific functional groups. However, these modifications can cause hydrolysis and thermal degradation of PEG coatings. A research group led by P.B.
Messersmith of Northwestern University has developed a biomimetic strategy to modify the biomaterial surfaces by incorporating 3,4-dihydroxyphenylalanine (DOPA) with PEG. DOPA, a catecholic amino acid formed by posttranslational modification of tyrosine, is a component of mussel adhesive proteins (MAPs). Known as one of the most notorious fouling organisms, mussels can adhere to wet surfaces by secreting this kind of protein glue. Messersmith's group used this idea and synthesized DOPA-containing peptidePEG conjugates to prepare nonadhesive/ nonfouling gold and titanium surfaces.

As reported in the April 9 issue of the Journal of the American Chemical Society, linear monomethoxy-terminated PEGs conjugated to a single DOPA residue (mPEG-DOPA) were synthesized as were PEGs conjugated to the N-terminus of Ala-Lys-Pro-Ser-Tyr-Hyp-Hyp-ThrDOPA-Lys (mPEG-MAPD), a decapeptide analogue of a protein found in the foot of mussel (Mytilus edulis) adhesive plaques. Surface analysis and cell culture experiments were carried out on the modified gold and titanium surfaceswhich are common implant materialsby absorbing mPEG-DOPA and mPEGMPAD in the solution.

$X$-ray photoelectron spectroscopy showed a significant ether (C-O) peak increase in mPEG-DOPA-treated gold substrates, compared with the unmodified materials, which, the researchers said, is typical of a surface-bound PEG polymer. The positive-ion time-of-flight secondary-ion mass spectroscopy spectrum revealed an increase in the presence of fragments corresponding to PEG absorption, which further confirmed formation of Au-DOPA complexes. A cell culture experiment was performed by culturing 3T3 fibroblasts on the surfaces for up to 14 days, and the ability of modified surfaces to resist cell attachment was examined by quantitative image analysis. Fluorescence microscopy images revealed that cell adhesion to mPEG-DOPA and mPEG-MAPD modified surfaces 